\title{
Vigilancia de tuberculosis en el Servicio de Salud Viña del Mar-Quillota entre los años 1999-2008. Estudio longitudinal de tendencia
}

JORGE RÍOS L.*, RICARDO ARIS S.**, AGATHA HERRERA U.** y MATÍAS RÍOS S.**

Surveillance of Tuberculosis in Health Service of Viña del Mar-Quillota, Chile from 1999 to 2008

Trend in Tuberculosis (TB) rates and association of factors affecting the outcome of this disease were assessed in the Health Service of Viña del Mar-Quillota, Chile from 1999 to 2008. Study of 1291 TB patients by the years 1999 to 2008. We evaluated incidence and mortality rate per 100,000 inhabitants, pulmonary / extrapulmonary ratio, presence of HIV, death and site of infection. It was found a decreasing trend in the incidence rate $\left(p<0.001 R^{2}=0.97\right)$ and association between the presence of HIV and case fatality rate $(p<0.001)$. It was not found association between site of disease and mortality rate $(p=0.3)$ nor a decreasing trend in mortality $(p=0.116)$ and pulmonary/extrapulmonary ratio $(p=0.194)$. There is a decreasing trend in the incidence rate of TB patients which reflects a wellfunctioning system of control and surveillance; the presence of HIV is associated with death because of this it should be considered in clinical management of TB.

Key words: Tuberculosis, epidemiology, surveillance, DOTS.

\section{Resumen}

Se evalúa la tendencia de las tasas de incidencia de tuberculosis (TBC) y de factores asociados al desenlace de esta enfermedad, en el Servicio de Salud Viña del Mar-Quillota durante los años 19992008. Estudio de 1.291 pacientes con TBC entre los años 1999-2008. Se evalúa tasa de incidencia y mortalidad por 100.000 habitantes, razón de casos pulmonares/extrapulmonares, presencia de VIH, tasa de letalidad y localización de la infección. Se observa una tendencia decreciente en la tasa de incidencia $\left(p<0,001 ; R^{2}=0,97\right)$ y asociación entre presencia de VIH y fallecer $(p<0,001)$. No se observa asociación entre localización de la enfermedad y fallecimiento $(p=0,3)$, ni tendencia decreciente en la tasa de mortalidad $(p=0,116)$ ni en la razón de casos pulmonares/extrapulmonares $(p=$ 0,194). La tendencia decreciente de la tasa de incidencia de TBC refleja un buen funcionamiento del sistema de control y vigilancia. La infección por VIH se asocia con mayor riesgo de muerte por lo que debe considerarse en el enfrentamiento clínico de la TBC.

Palabras clave: Tuberculosis, epidemiología, vigilancia, estrategia DOTS.

\section{Introducción}

La tuberculosis (TBC) es una de las tres principales amenazas mundiales de salud pública, junto al VIH/SIDA y a la malaria debido a su impacto en morbilidad, mortalidad, impacto socioeconómico, y al sufrimiento humano que causa $^{1}$. Durante gran parte del siglo XX, la TBC fue en Chile un problema de extrema gravedad, similar a lo observado en otros países de América Latina y en particular de la Región Andina. A partir de la creación del Servicio Nacional de Salud (1952) y de la implantación del actual Programa de Control de la TBC (1973) se registra un proce-

\footnotetext{
* Médico Asesor del Programa de Control de la Tuberculosis, Servicio de Salud Viña del Mar Quillota. Servicio de Tisiología Hospital de Peñablanca.

** Alumnos, Escuela de Medicina, Universidad de Valparaíso.
} 
so de sostenida reducción y control de la endemia en nuestro país². El reporte "Global tuberculosis control: surveillance, planning, financing" publicado en 2009 por la Organización Mundial de la Salud (OMS) indica que a nivel mundial, hubo 9,27 millones de casos incidentes estimados de tuberculosis en 2007. El número de casos ha aumentado en relación a los 9,24 millones de casos en 2006, 8,3 millones de casos en 2000 y 6,6 millones de casos en $1990^{3,5}$. Aunque el número total de casos incidentes de tuberculosis está aumentando en términos absolutos como resultado del crecimiento de la población, el número de casos por habitante está disminuyendo. Sin embargo, la tasa de disminución es menor al 1\% por año. Hubo una estimación de 13,7 millones de casos prevalentes de tuberculosis en 2007 (206 por 100.000 habitantes), una disminución en relación a los 13,9 millones de casos (210 por 100.000 habitantes) de $2006^{3}$. En Chile, la morbilidad por TBC en todas las localizaciones ha descendido de 52,2 por cien mil en 1989 a 18,4 en el año 2003. En un período similar (1989-2002), la mortalidad descendió de 5,9 a 2,0/100.000 habitantes. ${ }^{6}$

La inmunodepresión, la edad avanzada y la prescencia de comorbilidad, destacando la diabetes mellitus, son factores de riesgo frecuentes que determinan un mal pronóstico de la enfermedad ${ }^{7,8,11}$. Algunas causas de muerte como neumotórax, pericarditis, pleuritis, enfermedad metabólica, o incluso las infecciones oportunistas asociadas pueden ser prevenidas o curadas ${ }^{9}$. En relación a la infección por VIH en pacientes con TBC, la mortalidad a corto plazo sigue siendo elevada en los países en desarrollo y existe evidencia de que la proporción de casos de TBC intrapulmonar es mayor que en la población general ${ }^{10}$. Los enfermos a tratar de mayor relevancia en términos de salud pública son los portadores de TBC pulmonar con baciloscopías positivas al examen directo de expectoración debido a que poseen un mayor riesgo de contagio ${ }^{6}$.

En Chile, mientras en la población general la TBC está llegando a la etapa de eliminación, en los individuos VIH positivos la incidencia es todavía muy alta, pudiendo tener una frecuencia hasta 300 veces mayor ${ }^{12}$.

El DOTS (Directly Observed Treatment ShortCourse) es la estrategia recomendada internacionalmente para asegurar la curación de la TBC utilizada por la OMS.

Para frenar la diseminación de la enfermedad, esta estrategia se apoya en el diagnóstico precoz y la curación de los casos contagiosos y se basa en cinco principios clave que son: intervención organizada y sostenida; identificación temprana y precisa de casos; quimioterapia eficaz y fácil para el paciente; manejo eficaz de los medicamentos; y monitoreo basado en resultados ${ }^{2,13}$.

El Dr. Karel Styblo mostró que el tratamiento acortado era esencial para alcanzar tasas de curación adecuadas en el marco de un programa, verificó la necesidad del tratamiento directamente observado. La OMS comenzó a promover esta estrategia en 1991 y en 1994 produjo un Marco para el Control Eficaz de la Tuberculosis que describió claramente los componentes principales de lo que, a posteriori, se conoció como la estrategia DOTS. Este marco fue revisado y ampliado en el año 2002.

La estrategia DOTS fue aplicada en ochenta y dos países en 2003. Al final de este año, 77\% de la población mundial vivía en países que disponían de cobertura para esta estrategia. Los programas que utilizan el DOTS notificaron 3,7 millones del total de casos de TBC, de los cuales 1,8 millones eran nuevos casos bacilíferos, representando una tasa de detección de 45\%. En la región de América, 78\% de la población posee la cobertura de la estrategia DOTS con 142.409 nuevos casos de TBC, lo que representa una tasa de (16/100.000 habitantes) ${ }^{14,15}$.

El objetivo primario del presente estudio es evaluar en el Servicio de Salud Viña del Mar Quillota (SSVMQ) durante los años 1999-2008 la tendencia de: las tasas de incidencia de TBC, las tasas de mortalidad en pacientes tuberculosos y la proporción de casos de TBC intrapulmonar. Como objetivo secundario se plantea evaluar la asociación entre la mortalidad en pacientes tuberculosos y la presencia de infección por VIH y los casos de TBC intrapulmonar.

\section{Pacientes y Métodos}

Se realizó un estudio longitudinal de tendencia, para el cual se consideraron los casos de tuberculosis notificados entre los años 1999 a 2008, en el SSVMQ, incluyendo a la totalidad de los pacientes ingresados a tratamiento. Los datos fueron obtenidos de las tablas anuales de registros de TBC desarrolladas en el año de interés en el marco del programa nacional de control de la tuberculosis.

Los casos de TBC fueron diagnosticados/ confirmados con baciloscopía, cultivos, serología, biopsia, autopsia o antecedentes clínicos. Todos los casos fueron notificados y no existió subnotificación en atendidos por vía privada ya que la cobertura del programa es total y pacientes no notificados no reciben tratamiento. Todos 
los casos de pacientes con TBC que resultan ser VIH (+) son diagnosticados; así mismo los pacientes VIH (+) que son diagnosticados de TBC son notificados por los infectólogos tratantes. Se utilizó información del Instituto Nacional de Estadísticas (INE) de proyecciones poblacionales para el cálculo de tasas. Se calculó la tasa bruta de mortalidad general, ya que si bien al momento de fallecer un paciente se realiza una auditoría para determinar la causa de la muerte, su registro no está estandarizado por lo que la información disponible no permitió el cálculo de tasas de mortalidad específica según causa.

Las variables incluidas en el análisis fueron: año de notificación del caso, sexo, edad, localización de la enfermedad, tipo de confirmación diagnóstica, presencia de infección por VIH, tasa de incidencia de TBC, tasa de mortalidad por TBC y razón de casos pulmonares/extra-pulmonares. Las tasas fueron calculadas en base a información del INE y de acuerdo con proyecciones poblacionales.

Las variables fueron descritas mediante el cálculo de medianas, promedios y rangos en el caso de las variables continuas, y mediante porcentajes y frecuencias en el caso de las variables categóricas. Para evaluar tendencia en el tiempo se usó año de notificación como serie cronológica de datos y se utilizó la prueba de regresión de Prais-Winsten para mediciones correlacionales en el tiempo. El modelo de Prais-Winsten es un modelo de regresión lineal en el cual se deben cumplir los siguientes supuestos: las variables son dependientes del tiempo, los residuos están correlacionados y siguen un proceso autorregresivo de primer orden. Para explorar la asociación entre variables cualitativas categóricas, se utilizó la prueba exacta de Fisher y razón de odds para cuantificar la asociación. Para todas las pruebas estadísticas se estableció un nivel de significancia con un valor de p inferior a 0,05.

\section{Resultados}

\section{Descripción de los datos}

El número total de casos notificados de TBC en el SSVMQ en el periodo de estudio (19992008) fue 1.291, de los cuales 788 fueron hombres (61\%) y 504 fueron mujeres (39\%). El método diagnóstico mediante el cual se diagnosticó la infección se expone en la Tabla 1. En 917 pacientes la TBC fue de localización pulmonar $(71,6 \%)$ y en 363 (28,4\%) de localización extrapulmonar. La tasa de incidencia de TBC en el SSVMQ fue de 20 casos por 100.000 habitantes durante 1999 y 8 casos por 100.000 habitantes durante 2008 .
El promedio de edad de los pacientes notificados fue de 49,25 años con una desviación estándar de 18,76; la mediana de la edad fue de 49 años. La edad mínima fue menor a un año, y la máxima 92 años.

Al término del estudio, de los datos obtenidos se obtuvo que 79 pacientes fallecieron dentro del periodo de seguimiento y que 1.201 pacientes no fallecieron; la tasa de letalidad fue de $6,1 \%$. La tasa de mortalidad se encuentra entre 0,4 y 1,3 casos por 100.000 habitantes durante el periodo mencionado. En relación a la presencia de VIH, 59 pacientes fueron seropositivos (4,6\%) y 1.231 seronegativos (95,4\%).

La distribución del número de casos de TBC por año en el SSVMQ en presencia de VIH, según sexo, en ausencia de fallecimiento y en presencia de TBC pulmonar se grafica en la Figura 1.

\section{Inferencia estadística}

Se observó una tendencia decreciente en la tasa de incidencia de casos notificados por TBC en el decenio en el SSVMQ ( $p<0,001$ IC: $-1,53 ;-1,10)$. Al utilizar el modelo de predicción se estima que cada año, la tasa de incidencia de TBC decrece en 1,32 casos por cada 100.000 habitantes. El modelo de regresión explica en un $96,86 \%$ la variabilidad de la tendencia estudiada (Figura 2). Al evaluar la relación entre presencia de infección por VIH y mortalidad se observó una asociación positiva entre las variables: Los pacientes VIH positivos tienen 3,9 veces más riesgo de muerte en relación a los pacientes VIH negativos ( $\mathrm{p}<0,001$ IC: 1,72; 7,95) (Figura 3). No se observó una tendencia decreciente en la tasa de mortalidad $(\mathrm{p}=0,116)$ (Figura 4$)$ ni en la razón de casos pulmonares/extrapulmonares $(\mathrm{p}=$ $0,194)$ (Figura 5). No se observó asociación entre localización de la enfermedad y mortalidad $(\mathrm{p}=$ 0,3) (Figura 6).
Tabla 1. Distribución de casos notificados de TBC según confirmación diagnóstica. Servicio de Salud Viña del Mar-Quillota, 1999-2008

\begin{tabular}{|lcc|}
\hline Prueba diagnóstica & n & \% \\
\hline Baciloscopía & 611 & 48,03 \\
\hline Histopatología & 184 & 14,47 \\
Cultivo & 261 & 20,52 \\
Clínica & 204 & 16,04 \\
Serología & 12 & 0,94 \\
Total & 1.272 & 100,00 \\
\hline
\end{tabular}



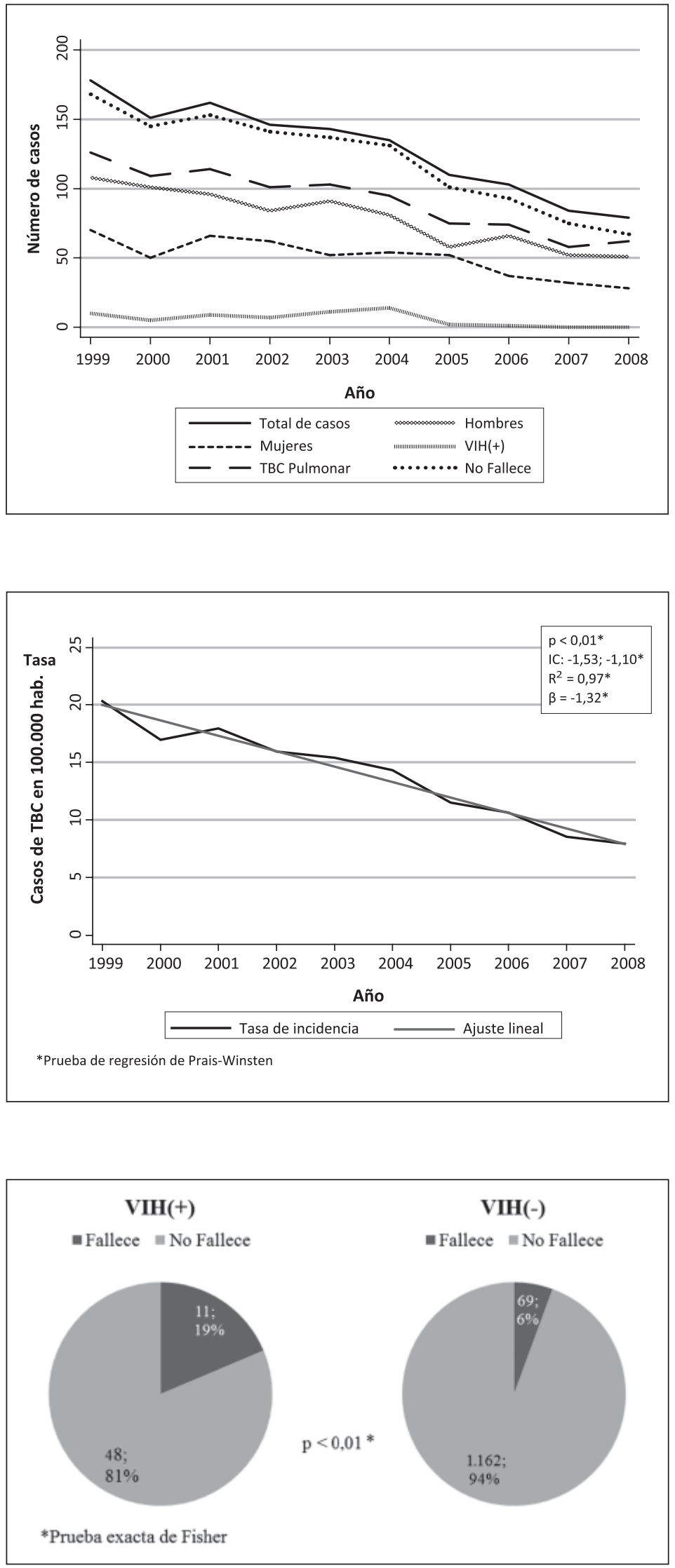

Figura 1. Número de casos de TBC por año en el Servicio de Salud Viña del Mar Quillota en presencia de VIH, según sexo, en ausencia de fallecimiento, en presencia de TBC pulmonar y casos totales entre los años 1999-2008.

Figura 2. Tasa de incidencia de TBC por año en 100.000 habitantes. SSVMQ 1999-2008.
Figura 3. Distribución de frecuencia y letalidad observada según antecedente de infección por VIH. SSVMQ 1999-2008. 

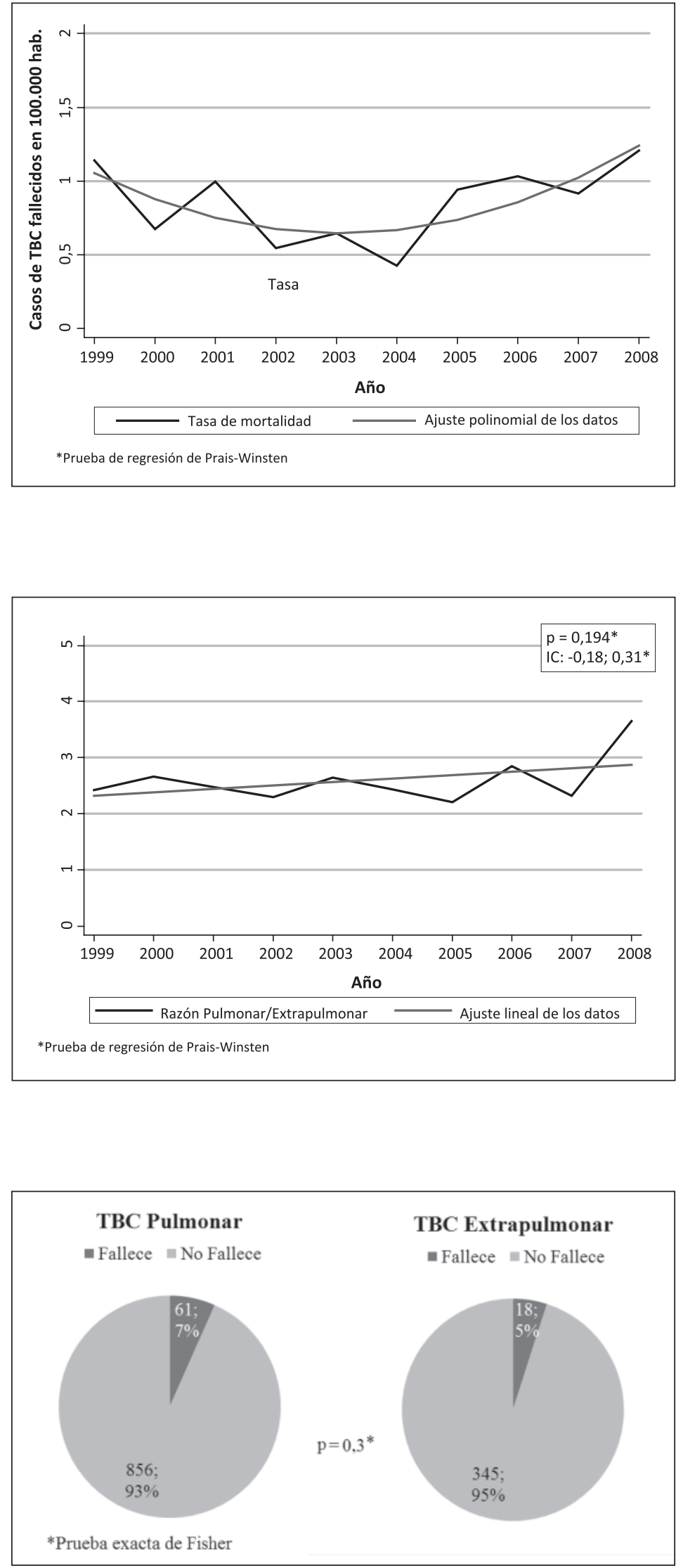

Figura 4. Tasa anual de mortalidad por TBC en 100.000 habitantes. SSVMQ 1999-2008.

Figura 5. Razón de casos de TBC pulmonares/extrapulmonares según año. SSVMQ 1999-2008.

Figura 6. Distribución de frecuencia y letalidad observada según localización de la infección. SSVMQ 1999-2008.

Rev Chil Enf Respir 2011; 27: 196-202 


\section{Discusión}

El presente estudio es el primero publicado a nivel de SSVMQ sobre incidencia de TBC y además, proporciona información que permite supervisar el programa de vigilancia y control de la TBC en el SSVMQ, contribuye al análisis epidemiológico de la enfermedad y es una herramienta útil de referencia que colaborará en la realización de estudios posteriores.

El estudio posee limitaciones debido al diseño retrospectivo del mismo: no fue posible controlar los factores de confusión a priori para evaluar asociación, ni es adecuado efectuar inferencia de los resultados a otras poblaciones; además, no se pudo obtener información detallada sobre tiempo de seguimiento medio de los pacientes, ni se presenta información en casos de fracaso de tratamiento (pérdida, abandono o resistencia). Se plantea la posibilidad de complementar esta línea investigativa diseñando un estudio de cohortes, para evaluar un análisis de sobrevida actuarial que permita elaborar una curva de Kaplan Meier, controlando la influencia de los factores de confusión señalados.

Se concluye que en la población estudiada en el Servicio de Salud Viña del Mar Quillota entre los años 1999-2008 existe una tendencia decreciente de la tasa de incidencia de TBC, reflejando posiblemente el adecuado funcionamiento del sistema de control y vigilancia, el cual está acorde con la situación global y concuerda con lo esperado, de acuerdo al plan de vigilancia y control de la TBC en Chile. ${ }^{3}$

Por otra parte, en contraste con lo esperado, ${ }^{3,4}$ no se puede afirmar que exista una tendencia decreciente de la tasa de mortalidad ni en la razón de casos pulmonares en relación a los extrapulmonares. Es necesario poner mayor énfasis en la prevención de complicaciones ${ }^{9}$, y en evitar la transmisión de esta patología, considerando especialmente a los pacientes con alto riesgo de contagio. ${ }^{6} \mathrm{Si}$ bien la incidencia va disminuyendo, la mortalidad no tiene un tendencia definida, lo cual puede explicarse teóricamente porque los casos son cada vez más complejos, difíciles de diagnosticar y en pacientes con patologías concomitantes y de mayor edad. No se espera que disminuya la letalidad (porcentaje de muertes por TBC dentro de los pacientes tuberculosos), pero se espera que la tasa de mortalidad disminuya cuando se reduzca de forma más significativa el número de casos.

La presencia de VIH se asocia a fallecer, situación que está explicada principalmente por su coexistencia con inmunosupresión y mayor riesgo de complicaciones, por lo que debe ser tomada en consideración tanto en el enfrentamiento clínico de la enfermedad, como en su evaluación en estudios posteriores. ${ }^{9,10}$ Además, este aumento de la letalidad en los pacientes VIH (+) concuerda con lo presentado por Villarroel et al ${ }^{12}$, por lo que la viabilidad y la eficacia de las estrategias actuales para el diagnóstico y tratamiento de la tuberculosis latente en pacientes seropositivos para el virus del VIH debieran ser revisadas.

Finalmente, contrario a lo esperado según los estudios revisados ${ }^{7,8}$, no se encontró asociación estadísticamente significativa entre localización pulmonar o extra-pulmonar de TBC y mortalidad en el SSVMQ entre los años 1999-2008.

\section{Agradecimientos}

Los autores agradecen a la Cátedra de Metodología de la Investigación, Universidad de Valparaíso, al Servicio de Salud Viña del Mar Quillota y al Instituto Nacional de Estadísticas, instituciones sin las cuales el estudio no hubiese podido llevarse a cabo.

\section{Bibliografia}

1.- VITORIA M, GRANICH R, GILKS CF, GUNNEBERG C, HOSSEINI M, WERE W, et al. The global fight against HIV/AIDS, tuberculosis, and malaria: current status and future perspectives. Am J Clin Pathol 2009; 131: 844-8.

2.- FARGA V. Hacia la erradicación de la tuberculosis.Rev Chil Enf Respir 2006; 22: 55-67.

3.- REPORT W. Global tuberculosis control: surveillance, planning,financing. 2009. Consultado el 10.09.2009, de http://www.who.int/tb/publications/global_report/2009/ key_points_download/en/index.html

4.- REPORT EW-ES. Tuberculosis surveillance in Europe 2009. Consultado el 10.09.2009, de http://ecdc.europa. eu/en/publications/Publications/1103_TB_SUR_2009. pdf.

5.- Management of Tuberculosis in United States. N Engl J Med 2009, 345.

6.- MINISTERIO DE SALUD C. Programa Nacional para el Control de la Tuberculosis. Norma General Técnica 2005; No 82.

7.- GARCÍA-FERNÁNDEZ C, VERA LÓPEZ I, JAEN HERREROS F, SANZ-GALLARDO MI, PALENQUE MATAIX E, DE JUANES PARDO JR. Tuberculosis in the elderly in a general hospital in the Region of Madrid, 1994-2003. An Med Interna 2006;23:161-5.

8.- HOHEISEL G, HAGERT-WINKLER A, WINKLER J, 
KAHN T, RODLOFF AC,WIRTZ H, et al Pulmonary and pleural tuberculosis in the elderly. Med Klin (Munich) 2009;104:772-9.

9.- NGO AT, DUC NH, LAN NH, MAYNART M, MAYAUD C, QUY TH. Mechanisms and causes of death in 143 Vietnamese HIV-infected patients hospitalized for tuberculosis. Rev Pneumol Clin 2007;63:139-46.

10.- ELHARTI E, ZIDOUH A, MENGAD R, BENNANI O, ELAOUAD R. Monitoring HIV through sentinel surveillance in Morocco. East Mediterr Health J 2002;8:141-9.

11.- WENG SF, HSU CH, LIRN ML, HUANG CL. Extrapulmonary tuberculosis: a study comparing diabetic and nondiabetic patients. Exp Clin Endocrinol Diabetes 2009;117:305-7

12.- VILLARROEL L, RABAGLIATI R, BALCELLS M, KARZULOVIC L, PÉREZ C. Tuberculosis en individuos con infección por VIH en Chile: Estudio de prevalencia e impacto sobre mortalidad. Rev Méd Chile 2008,136:578-86.

13.- What is DOTS? A guide to understanding the WHOrecommended TB control strategy known as DOTS. Geneva, World Health Organization, 1999 (document WHO/CDS/CPC/ TB/99.270). Consultado el 10.09.2009, de http://whqlibdoc.who.int/hq/1999/ WHO_CDS_CPC_TB_99.270.pdf

14.- ORGANIZACIÓN PANAMERICANA DE LA SALUD. Avances en el control de la tuberculosis en la Región de las Américas: 1995- 2003. Tuberculosis 2004 marzo; 7:1-3.

15.- VENDRAMINI SHF, VILLA TZS, SANTOS MLSG, GAZETTA C E. Aspectos epidemiológicos actuales de la tuberculosis y el impacto de la estrategia dots en el control de la enfermedad. Rev Latino-am Enfermagem 2007; 15: 171-3.

Correspondencia a:

Dr. Jorge Ríos Lagos

Servicio de Tisiología, Hospital de Peñablanca.

E-mail: tisiologia@yahoo.es

Ricardo Javier Aris Serey.

Casilla 151, Quilpué, Chile.

E-mail: rjariss@gmail.com 